\title{
Thalassemia Major and related Dentomaxillofacial Complications: Clinical and Radiographic Overview with Reference to Dental Care
}

\author{
Faiez N Hattab
}

\begin{abstract}
Introduction: Thalassemia is one of the most common genetic disorders worldwide and presents public health and social challenges in areas of high incidence. Thalassemia major (TM) type is associated with most severe clinical changes.
\end{abstract}

Aim: To evaluate dental and oromaxillofacial features of TM.

Materials and methods: The sample consisted of 54 children and adolescents with TM, 31 males and 23 females, aged 5.5 to 18.3 years (mean age $11.6 \pm 3.2$ years) and thalassemia-free controls. They were examined for dental caries, oral hygiene, periodontal status, orofacial features, tooth size and dental arch dimensions, dental development, and physical pattern. Clinical, radiographical, and odontometric assessments were carried out.

Results: Thalassemia major patients were at high risk of dental caries. The average decayed, missing, filled tooth (DMFT) value was 6.26 for 15 -year-olds compared with 4.84 in the control group $(p<0.001)$. Poor oral hygiene and gingivitis were observed in 61.1 and $92.2 \%$ of the thalassemic patients respectively. Clinical orofacial features of TM include frontal bossing, saddle nose, and maxillary protrusion, giving a "chipmunk"-like face. Lip incompetence, dental discoloration, and pallor oral mucosa have been noted. Radiographical examination showed the following: thickened frontal bone, thinned mandibular cortex, and maxillary sinus hypoplasia. Class II malocclusion, significant reduction in tooth size and dental arch dimensions, and delay in dental development were evident. Growth retardation was present in $75.9 \%$ of the patients.

Conclusion: Thalassemia major produces a variety of orofacial and systemic complications that increase with age. Early diagnosis and management allow more favorable prognosis and minimize complication.

Clinical significance: Dental practitioners, especially working in multiracial communities, require understanding of the complications and management of the disease.

Keywords: Dental care, Dental development, Orofacial features, Physical growth, Thalassemia major.

How to cite this article: Hattab FN. Thalassemia Major and related Dentomaxillofacial Complications: Clinical and Radiographic Overview with Reference to Dental Care. Int J Experiment Dent Sci 2017;6(2):95-104.

\section{Visiting Professor \\ Essen, Düsseldorf, Germany}

Corresponding Author: Faiez N Hattab, Visiting Professor Essen, Düsseldorf, Germany, e-mail: f_hattab@hotmail.com

\section{Source of support: $\mathrm{Nil}$}

Conflict of interest: None

\section{INTRODUCTION}

Thalassemia refers to a group of inherited hemolytic anemic disorders that involve defects in the synthesis of hemoglobin alpha- or beta-polypeptide chains, known as alpha- or beta-thalassemia. Reduced synthesis of one of the two globin polypeptides leads to decreased hemoglobin $(\mathrm{Hb})$ production, resulting in hypochromic microcytic anemia associated with erythrocyte dysplasia and destruction. Thalassemia comprises several subtypes of diverse clinical characteristics. Beta-thalassemias can be classified into TM, thalassemia intermedia, and thalassemia minor, with other variants. Due to its genetic heterogeneity and clinical and hematologic variability, thalassemia is classified as homozygous, heterozygous, or compound heterozygous. Homozygous beta-thalassemia (also known as TM, Cooley anemia, or Mediterranean anemia) is associated with the most severe signs and symptoms among other types of thalassemia. The manifestations of the condition are modulated by several genetic, racial, and environmental factors. More than 200 beta-thalassemia mutations have been reported to date. ${ }^{1}$ Most thalassemia types are inherited as autosomal recessive traits, apart from the rare dominant forms.

\section{EPIDEMIOLOGY}

Thalassemia is one of the most common genetic disorders worldwide and presents major public health and social challenges in areas of high incidence. It has estimated that about $3 \%$ of the world's populations carry the thalassemia gene. Males and females are equally affected. The frequency of this disorder varies considerably with geographic locations and racial groups. It is most common among individuals of Mediterranean descent, particularly those living in southern Italy, Greece, and Cyprus, where prevalence is 10 to $15 \%{ }^{1,2}$ The disorder has also been described in Africa, Southeast Asia, Arab countries, Turkey, and Islamic Republic of Iran, with frequency in those regions ranging from 2.5 to $10 \%$. It has been estimated that about $1.5 \%$ of the 
global population (80-90 million people) are carriers of beta-thalassemia, the great majority in the developing world. ${ }^{2}$ Due to migration and intermarriage between different ethnic groups, thalassemia has become a disease of international interest.

\section{PATHOPHYSIOLOGY}

Anemia, caused by the disease, stimulates bone marrow to increase production of erythrocytes (via erythropoietin) to maintain adequate oxygenation; however, erythrocyte production is abnormal. The process is called "ineffective erythropoiesis," causing hypertrophy and expansion of the erythroid marrow cavity, impairs bone growth, and increases bone resorption. As a result, osteopenia or osteoporosis, decreased mineralization, and decreased bone-forming sites are common findings. ${ }^{3-5}$ In addition, the expansion of bone marrow can cause mechanical interruption of bone, cortical thinning, bone distortion, and increased fragility. With time, massive erythropoiesis (formation of erythrocytes) within bone marrow cavities of the skull bones, facial bones, and ribs leads to bone expansion (up 25-30 times). ${ }^{1}$ Ineffective erythropoiesis leads to excessive supply of iron and iron overload (hemosiderosis). Iron overload also occurs as a result of severe chronic hemolysis of erythrocytes, regular blood transfusions, decrease in $\mathrm{Hb}$ production, and the increased absorption of dietary iron in response to the severely anemic state. Iron overload progressively develops clinical manifestations mainly in the heart (myocardial siderosis), liver (fibrosis and cirrhosis), and endocrine glands (diabetes mellitus, hypogonadism, and insufficiency of the parathyroid, thyroid, pituitary, and adrenal glands). ${ }^{6-8}$

\section{CLINICAL DIAGNOSIS}

Thalassemia major is a life-threatening condition that commonly manifests during early infancy, after which progressive pallor, severe anemia, and failure to thrive are common. Children with TM often develop feeding problems, recurrent fever, bleeding tendencies (especially epistaxis), susceptibility to infection, pathologic fractures of long bones and vertebrae, endocrine abnormalities, jaundice, splenomegaly, lack of sexual maturation, and growth retardation. ${ }^{3,8-10}$ The Hb level may be as low as 3 to $5 \mathrm{gm} / \mathrm{dL}$ when a child with TM becomes symptomatic. ${ }^{10}$ The manifestations of TM depend on the severity of anemia, the patient's age, duration of the clinical symptoms, the timing and frequency of blood transfusion, age of initiation of iron chelation therapy, and splenectomy. In response to the underlying anemia, the gross bone marrow expansion due to ineffective erythropoiesis can cause mechanical interruption of bone formation, cortical thinning, bone distortion, and increased fragility. Hormonal abnormalities appear to underlie the altered bone turnover observed in TM. ${ }^{6,7,11}$ These changes give most of the characteristic features of TM, including typical skeletal and oromaxillofacial deformities.

The existing literatures regarding the orofacial manifestation of TM are limited mainly to case reports and short studies, and only few large studies have been undertaken. Little data on the dental management of the disease are available. The aim of the present overview was to evaluate changes in dental and orofacial related to TM. Guidance on the management and provision of optimal dental care is also presented. It is hoped that this review will alert dental practitioners, especially working in multiracial communities to understand the nature of this disorder, its implication on the oral health and dental care. Ethical approval for the study and consent from parents were obtained.

\section{MATERIALS AND METHODS}

A total of 54 children and adolescents with TM, 31 males and 23 females aged 5.5 to 18.3 years, were selected. Mean age \pm standard deviation (SD) was $11.6 \pm 3.2$ years. A thalassemia-free group matched by age and gender served as a control group. Clinical, radiographical, and odontometric examinations were carried out to assess changes caused by this disorder. The subjects were examined for dental caries, oral hygiene, periodontal status, orofacial features, tooth size and dental arch dimensions, dental development, and physical pattern. Measurements of mesiodistal crown diameter (MD), also called tooth crown size, or tooth size, and dental arch dimensions were made on the dental casts of the participants using electronic digital sliding caliper reading to $0.01 \mathrm{~mm}$. For each maxillary and mandibular permanent tooth, MD was registered by measuring the greatest distance between proximal surfaces of the crown. Maxillary and mandibular dental arch dimensions were measured for depth, length, and interdental width (Fig. 1).

Arch perimeters were measured using a brass wire contoured along the line of occlusion and then straightened out. Linear and angular measurements of the mandible were obtained from tracing the panoramic radiographs. Dental development, defined from first appearance of calcified points to apex closure, was assessed according to the method of Demirjian et al. ${ }^{12}$ Student's $t$-test was used to compare the means between thalassemic and control groups. Pearson's correlation coefficients (r) were employed to describe correlations between dental development and chronological age (CA). 

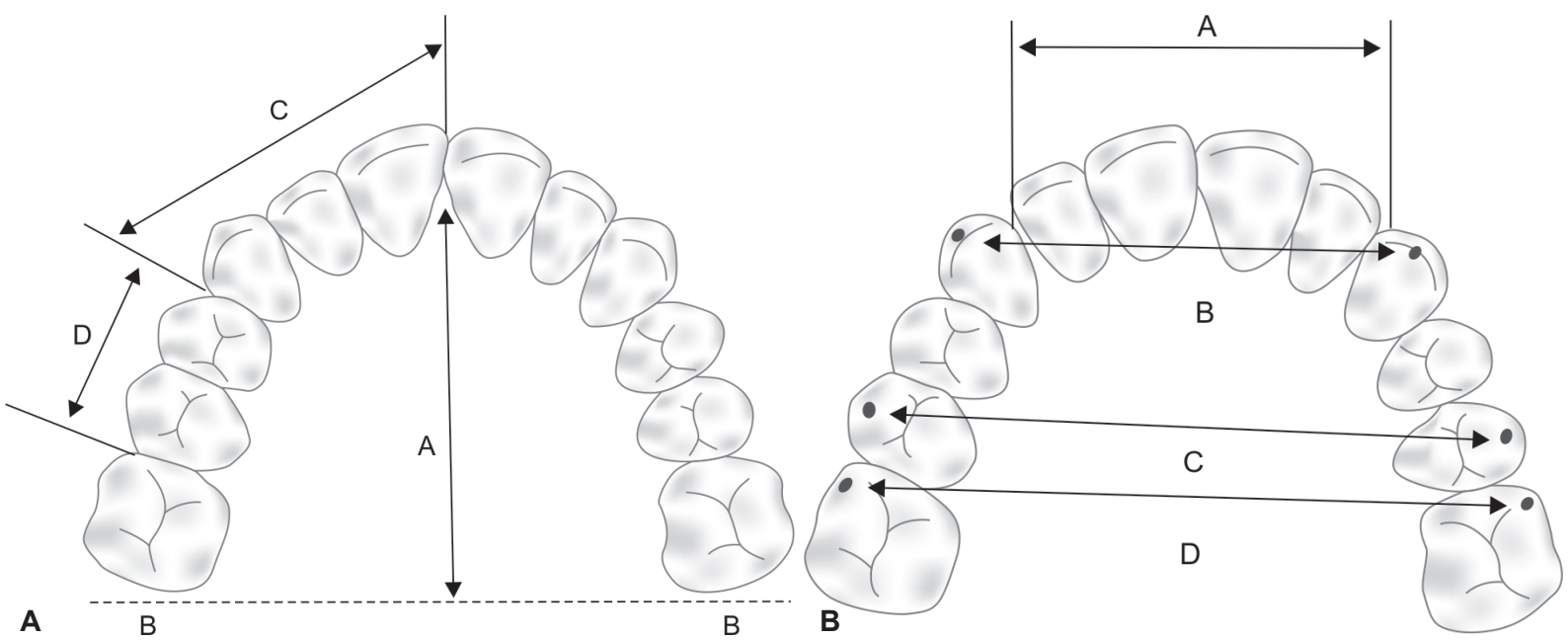

Figs $1 \mathrm{~A}$ and B: (A) Dental arch dimensions measurement. A: arch depth; B: arch perimeter; C: anterior arch length; D: posterior arch length. (B) Dental arch width measurements: A: interincisor width; B: intercanine width; C: interpremolar width; D: intermolar width ${ }^{18}$
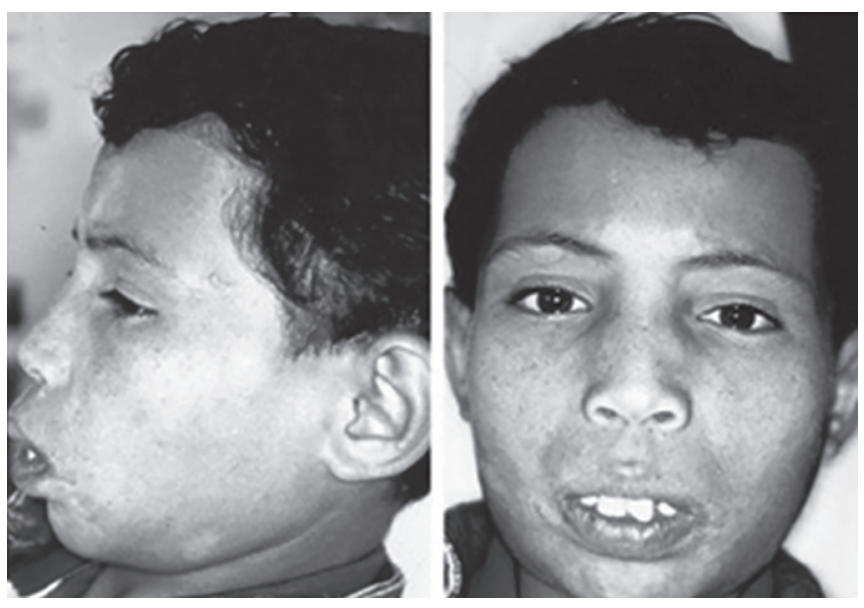

Fig. 2: Profile view of a 13-year-old boy with TM showing typical facial features of TM, characterized by frontal bossing, bulging cheekbone, saddle nose, and protrusive premaxilla (chipmunk face)

\section{RESULTS}

\section{Dental and Oromaxillofacial Features in TM}

The typical oromaxillofacial deformities observed in TM patients are frontal bossing, prominent cheek (malar) bones, saddle nose, maxillary protrusion, flaring of the maxillary anterior teeth, lip incompetence, and malocclusion. These changes give a distinctive "chipmunk"-like appearance (Fig. 2).

Of the patients examined, $33 \%$ had almost normal appearance, $26 \%$ (grade I: slight depression of the nose, puffiness of the eyelids with no maxillary overgrowth), $24 \%$ (grade II: mild maxillary overgrowth and slight bulging of the frontal and cheek bones), and 16.7\% (grade III: "chipmunk" facies).

\section{Dental Caries}

Although there is clear evidence that dental caries is a multifactorial infectious disease with many contributory environmental factors, there is also strong evidence for a genetic component in the etiology of this disease. The mean DMFT value was 5.82 for 6 to 9 years old. The DMFT value was 6.57 for 12 to 14 years and 5.95 for 15 to 18 years old. The caries prevalence in TM patients was significantly higher than the control group, with an average DMFT for 15 years old was $6.26 \pm 2.24( \pm \mathrm{SD})$ compared with DMFT $4.84 \pm 2.01( \pm \mathrm{SD})$ for the control group $(\mathrm{p}<0.001)$. Only $17.4 \%$ of the children aged 6 to 9 years and $21.4 \%$ of 12 to 18 years old were caries-free. ${ }^{13}$

\section{Periodontal Status and Oral Hygiene}

Thalassemia major patients showed a higher plaque index and gingival index (GI) scores compared with the control group. The mean PI score in thalassemic group was $1.66 \pm$ 0.51 vs $1.55 \pm 0.65$ in the control group. The GI showed that $49.2 \%$ of the thalassemic patients had mild gingivitis (no bleeding on probing), $34.7 \%$ moderate gingivitis (bleeding on probing), and $8.3 \%$ severe gingivitis (spontaneous bleeding). Only $7.8 \%$ showed no sign of gingivitis vs $25.2 \%$ in the controls. Pathologic gingival sulcus depths in thalassemic patients found more frequent than in controls. About two-thirds of the patients had poor oral hygiene and $32.5 \%$ exhibited supra- and subgingival calculus deposit. ${ }^{14}$

\section{Tooth and Mucosal Discoloration}

Due to chronic jaundice associated with thalassemia, the incorporation of blood pigment bilirubin, degraded product of $\mathrm{Hb}$, in the dentinal tubules during tooth formation results in yellow discoloration of teeth. ${ }^{15}$ Tooth discoloration and pallor oral mucosa has been found in 44.4 and $38.9 \%$ respectively, in TM patients (Table 1).

\section{Tooth Crown Size and Tooth Size Ratio}

All means of MD in thalassemic males and females were smaller than their controls, with 20 of the 24 comparisons 
Table 1: Prevalence of clinical TM features in different population groups

\begin{tabular}{llll}
\hline TM sample & Jordanian & Iranian & Indian \\
\hline Sample size & $54^{14}$ & $119^{21}$ & $72^{22}$ \\
Feature, \% (n) & & & \\
Frontal bossing & $61.1(33)$ & - & - \\
Saddle nose & $59.2(32)$ & $34.0(40)$ & $56.9(41)$ \\
Lip incompetence & $51.8(28)$ & $47.9(57)$ & $80.6(58)$ \\
Discolored teeth & $44.4(24)$ & - & - \\
Dental and jaw pain & $40.7(22)$ & - & - \\
Pallor oral mucosa & $38.9(21)$ & $26.9(32)$ & $22.2(16)$ \\
Headache & $29.6(16)$ & - & - \\
Increased overjet & $25.9(14)$ & - & - \\
Maxillary protrusion & $24.1(13)$ & $60.5(72)$ & - \\
Chipmunk faces & $16.7(9)$ & - & $41.7(30)$ \\
Nasal airway problem & $16.7(9)$ & - & - \\
Lower lip paresthesia & $13.0(7)$ & - & - \\
Parotid gland enlargement & $5.6(3)$ & - & - \\
\hline
\end{tabular}

being statistically significant (ranging from $p<0.05$ to $\mathrm{p}<0.001$ ). In both thalassemic and control groups, males exhibited significantly larger MD than females in most instances. Canines displayed the most sexual dimorphic teeth in the dentition. Lateral incisors showed the greatest variable teeth. ${ }^{16,17}$ The tooth size ratio describes the discrepancy between the sums of MD of mandibular relative to the maxillary teeth. The anterior and overall ratios (sexes pooled) in thalassemic group were 79.1 and 92.0 respectively. The corresponding ratios of the control group were 79.4 and 92.4 respectively. The differences in tooth size ratio between thalassemic and control group were not statistically significant. ${ }^{17}$

\section{Dental Arches Dimensions}

Measurements showed that the segmental arch lengths in the maxilla and mandible of thalassemic group were reduced by an average of 2.59 and $2.55 \mathrm{~mm}$ respectively, compared with the control group. The mean maxillary and mandibular arch depths (lengths) were shorter by 3.21 and $2.63 \mathrm{~mm}$ respectively, relative to the controls $(\mathrm{p}<0.001)$. All arch widths in thalassemic patients were significantly reduced by an average ranging from 1.33 to $1.90 \mathrm{~mm}$ in the maxilla and 1.37 to $1.77 \mathrm{~mm}$ in the mandible. The mean maxillary and mandibular arch perimeters were reduced by 3.91 and $3.44 \mathrm{~mm}$ respectively, in the thalassemic group relative to the controls $(\mathrm{p}<0.001) .{ }^{18}$

\section{Occlusion}

Lateral cephalometric radiographs showed that almost all thalassemic patients had a class II skeletal base relationship. ${ }^{19}$ The protrusive premaxilla frequently results in an increased overjet, spacing, and forward drifting

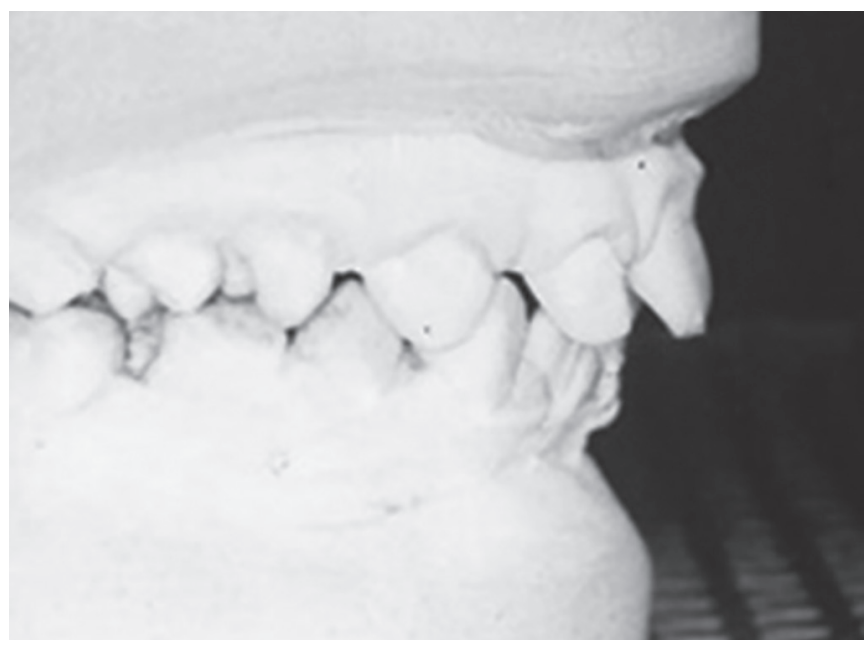

Fig. 3: Dental cast of TM patient showing maxillary protrusion, increased overjet, flaring of the maxillary anterior teeth, spacing of teeth, and malocclusion

of the maxillary anterior teeth with varying degrees of malocclusion (Fig. 3).

\section{Dental Development and Physical Growth}

Assessment of dental development using panoramic radiographs showed that $79.5 \%$ of thalassemic patients had a delay of dental development, relative to CA. A delay of 1.12 years in males and 0.81 years in females has been found. The association between dental age (DA) and CA was stronger than that between DA and body growth $(\mathrm{r}=0.87$ vs 0.58$) .{ }^{20}$ Growth retardation was present in $75.9 \%$ of the patients. Height less than the third percentile has been reported in $41.9 \%$ of males and $34.8 \%$ of females. Growth retardation becomes more evident in patients older than 10 years. The estimated body mass index (BMI) of thalassemic patients was $16.5 \pm 2.2 \mathrm{~kg} / \mathrm{m}^{2}$ compared with 18.5 to $24.9 \mathrm{~kg} / \mathrm{m}^{2}$ for healthy weight. Moreover, BMI was less than the 10 th percentile in $21.6 \%$ of younger than 10 years $v s 37.2 \%$ of that older than 10 years. ${ }^{20}$

\section{Frequency of Orofacial Features}

The frequency of dental and oromaxillofacial features of the present TM patients compared with other population groups is shown in Table 1.

\section{Radiographic Features}

Panoramic and lateral cephalometric radiographs have been used to evaluate dental, mandibular, and craniofacial abnormalities in TM patients. The findings are presented in Table 2 and depicted in Figures 4 and 5. The majority of patients had thickened frontal bone $(66.7 \%)$ and thinned inferior border of the mandible (mandibular cortex, $64.6 \%:<3.3 \mathrm{~mm}$ ). Thinning of the mandibular 
Table 2: Panoramic $(n=48)$ and lateral cephalometric $(n=33)$ radiographs showing the prevalence of oromaxillofacial abnormalities in thalassemic groups

\begin{tabular}{lll}
\hline Feature & Number & Percentage \\
\hline Thickened frontal bone* $^{*}$ & 22 & 66.7 \\
Thinned mandibular cortex** $^{*}$ & 31 & 64.6 \\
Maxillary sinus hypoplasia* $^{*}$ & 14 & 42.4 \\
Faint lamina dura** $^{*}$ & 19 & 39.6 \\
Enlarged marrow spaces** & 18 & 37.5 \\
Widened diploic spaces* $^{*}$ & 12 & 36.4 \\
Short spiky roots $^{* *}$ & 16 & 33.3 \\
"Hair-on-end" calvarium* & 2 & 6.1 \\
\hline
\end{tabular}

*Lateral radiographs; ${ }^{* *}$ Panoramic radiographs ${ }^{14}$

cortex was found accompanied with faint or absence of the inferior alveolar canal (mandibular canal) borders. Enlarged marrow spaces, faint lamina dura, and small maxillary sinus were noted between 37.5 and $42.4 \%$ of the patients' radiographs. Short spiky roots and widened diploic spaces have been observed in 33.3 and $36.4 \%$ of the patients' radiographs.

The alveolar bone showed generalized rarefaction due to enlarged marrow spaces and altered trabecular pattern, characterized by the apparent coarsening of the some trabeculae and the blurring or disappearance of others producing "chicken-wire" appearance. Hair-on-end skull appearance is relatively uncommon radiographic findings. It represents an alternating opaque trabeculae and diploic (spongy) radiolucent marrow oriented perpendicular to the inner and outer tables of the skull, giving appearance of long, thin vertical striations in a radial pattern.

\section{Radiographic Measurements}

Linear and angular measurements of the mandible obtained from tracing the panoramic radiographs

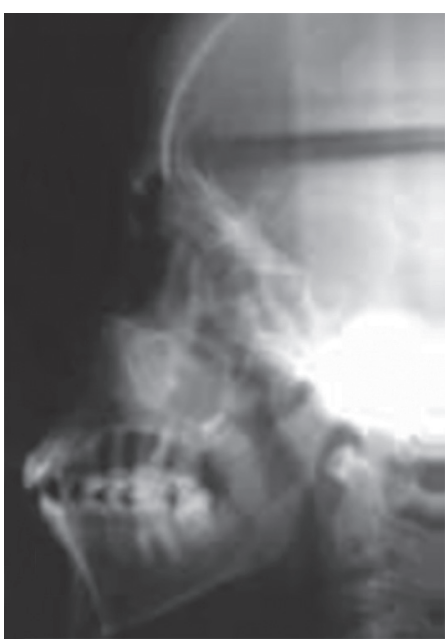

Fig. 4: Cephalometric radiograph of a 15 -year-old boy with TM disclosing thickened frontal bone, thinned inferior border of the mandible, and prominent premaxilla. Note small maxillary sinus and widened diploic spaces in the frontal bone revealed significant reduction in the ramus length and width of TM patients compared with the controls. The mean ramus length and width were reduced by 3.2 and $1.8 \mathrm{~mm}$ respectively, compared with the control group. The mean intercondylar distance was reduced by $6.9 \mathrm{~mm}$. The mean gonial angle $\left(127.3^{\circ}\right)$ was significantly larger in thalassemic than that in controls.

\section{DISCUSSION}

Thalassemia major is a serious medical, social, and psychological problem. The course of illness depends on early and regular blood transfusion, iron chelation, and other therapeutic facilities. When such treatments are given, many patients in developed countries survive to the fifth decade of life. ${ }^{6,23}$ Patients with optimally treated thalassemia can now enjoy a near-normal life and lifestyle, and experience regular physical and emotional development from childhood to adulthood. It is now universally recognized that thalassemia, like other chronic diseases, has important psychological implications. Medical advances continue to improve the life expectancy and quality of life further for those living with thalassemia.

\section{Dental and Orofacial Features}

The majority of orofacial manifestations are in response to the severe hemolytic anemia, chronic hypoxia, and ineffective erythropoiesis, resulting in massive bone marrow hyperplasia and expansion of the marrow cavity. These changes are mainly manifested as follows: (1) Bossing of the frontal bone, prominence of the malar bone, and maxillary protrusion; (2) thinning of mandibular inferior cortex, faint or absence mandibular canal; (3) enlarged

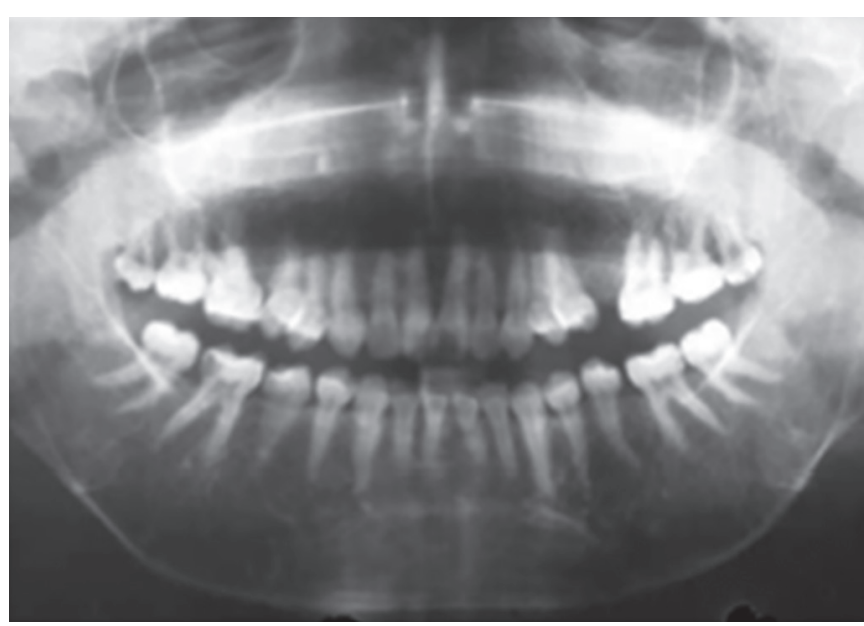

Fig. 5: Panoramic radiograph of TM patient demonstrating thinned mandibular inferior cortex, enlarged bone marrow spaces, short spiky roots of the mandibular molars, thin lamina dura, indiscernible border of the mandibular canal 
marrow spaces, altered (reduced) trabecular pattern of the mandible; (4) partially obliterated maxillary sinus due to delayed pneumatization. A skull radiograph may show a prominent "hair-on-end" appearance of the calvaria. Abnormalities in orofacial and cranial expressions became more pronounced with increasing age.

Review of recent studies showed that the normal mandibular cortex width varies between 3.24 and $6.55 \mathrm{~mm}^{24,25}$ (mean $\pm \mathrm{SD}=4.98 \pm 0.87 \mathrm{~mm}$, number of studies: 14), with threshold value for osteoporosis ranging between 3.1 and $4.5 \mathrm{~mm}$ (mean $\pm \mathrm{SD}=3.38 \pm 0.75 \mathrm{~mm}$ ). The present study indicates that two-thirds of the TM patients had thinning of the mandibular cortices $(<3.3 \mathrm{~mm})$. Earlier report on Jordanian TM patients showed contradiction in the given values of mandibular cortex thickness by stating that: "In the majority of patients $(60 \%)$, the thickness of the inferior border was between $1 \mathrm{~mm}$ and $2.5 \mathrm{~mm}$. . In the same paragraph added: "Thirty-five patients (70\%) were between $4.5 \mathrm{~mm}$ and $6 \mathrm{~mm} .{ }^{\prime 26}$ Further studies are needed to determine the mandibular cortex thickness among different thalassemic age groups.

Incompetency of the lips is due to maxillary protrusion and proclined maxillary anterior teeth, preventing complete closure of the lips. This leads to mouth breathing habit and mouth dryness that may contribute to the etiology of dental caries and periodontal disease. Pallor of the oral mucosa may arise from the chronic anemic status of the patients. Thalassemic patients also experience dental and jaw pain, transitory headache, and lip paresthesia. The lack of earlier studies on these symptoms in thalassemic patients precludes direct comparisons with the present findings. Painful swelling of the parotid glands and reduced salivary secretion may result from iron deposition. Increased oral Candida albicans colonization has been noted in TM patients. $^{27,28}$

\section{Dental Caries}

The high incidence of dental caries in thalassemic patients is well documented in different population groups. . $^{13,29,30}$ In a study on 47 Italian TM patients, Leonardi et $\mathrm{al}^{31}$ found that $90 \%$ of males and $66 \%$ of females had caries. A high caries score (DMFT: 5.12) has also been reported in 60 Italian patients where only $8 \%$ were caries-free. ${ }^{32}$ These findings are in agreement with our results on Jordanian patients, which showed that $78.6 \%$ of the patients aged 12 to 18 years had caries. ${ }^{13}$ The World Health Organization described the prevalence of caries (DMFT) above 5.6 as very high. ${ }^{33}$ The reasons for caries risk in TM could be attributed to poor oral hygiene, ${ }^{13}$ reduced salivary flow rate, ${ }^{13}$ lower concentration of salivary immunoglobulin, ${ }^{34}$ higher levels of salivary Streptococcus mutans, ${ }^{35}$ improper dietary habits, lack of dental awareness, and neglected dental care.

Thalassemic patients show higher occurrence of periodontal disease relative to thalassemia-free controls. ${ }^{14,29,30}$ Recent study showed that the level of salivary aspartate aminotransferase and alanine aminotransferase enzymes in TM patients was much higher than in the healthy control group. ${ }^{28}$ These enzymes are proposed as markers for periodontal disease activity. Systemic factors including lower resistance to infection, nutritional deficiency, chronic anemia, and hormonal imbalance contributes to the complexity of etiology. Current evidence supports an association of osteoporosis, a common feature of TM, with the onset and progression of periodontal disease. ${ }^{36}$

\section{Tooth Crown Size and Dental Arches Dimensions}

Tooth crown size exhibits a continuous range of variation among individuals and between different populations, a reflection of complex interaction between a variety of genetic, racial, and environmental factors. Several maternal conditions and gestational variables have influence on tooth size, including diabetes, hypothyroidism, hypertension, birth weight, and length. ${ }^{37}$ The reduced tooth size may render the dentoalveolar bone housing the teeth to be more deficient. The shorter dental arches depth and the narrower arch width in TM patients could be a reflection of general growth retardation and skeletal changes in thalassemic patients. Local factors that influence dental arch morphology are eruption, position, number, and size of teeth.

\section{OCCLUSION}

Thalassemic patients may develop malocclusion subsequent to maxillary protrusion and mandibular atrophy. The less protruded is mandible than maxilla, apparently because the dense mandibular cortical layer resists expansion. There is a variation regarding the frequency and type of malocclusion in TM patients as determined clinically, radiographically, or using dental casts. Review of literature showed that the incidence of class II malocclusion varies considerably. One of the explanations for this variation could be derived from Scutellari et $\mathrm{al}^{38}$ who demonstrated that all TM patients being treated with low transfusion regimes had dental and/or skeletal class II malocclusion vs $40.7 \%$ of the adequately transfused patients had malocclusion.

\section{Dental Development}

The development of the dentition is considered an integral part of craniofacial growth. Dental development or 
maturity, expressed as DA, is one of the most reliable indicators of CA and widely used in forensic and legal dentistry. A significant delay in dental development among TM patients has been documented. ${ }^{20}$ Further, there was a significant correlation between the extent of this delay and CA, but not between dental maturity and physical development. This is in line with the findings that dental development is less influenced than somatic development by environmental factors. ${ }^{39}$ The significant delay in the dental development, reduction in tooth crown size, and reduced dental arches dimensions in TM could be attributed to the genetic, hormonal, and pathological entities underlying the disease.

\section{Physical Growth}

The cause of growth retardation in TM patients is multifactorial, including chronic anemia and hypoxia, iron overload, racial factors, endocrinopathies, and low socioeconomic status. ${ }^{3,8,9}$ A marked delay in physical growth of thalassemic patients has been documented in different population groups. Hattab ${ }^{20}$ reported growth retardation in $75.9 \%$ of Jordanian children and adolescents with TM. Their height found less than the third percentile in $41.9 \%$ of males and $34.8 \%$ of females. The low BMI of TM patients indicate that the patients are underweight. Borgna-Pignatti et $\mathrm{al}^{9}$ reported that among 250 Italian adolescents with TM, $62 \%$ of males and 35\% of females had short stature. Kattamis et $\mathrm{al}^{40}$ found that $21.7 \%$ of males and $13 \%$ of females had growth retardation among a group of 405 Greeks with TM; the highest incidence of growth retardation was among those aged 15 to 20 years. A study of 68 Chinese children with TM in Hong Kong showed that $75 \%$ of girls and $62 \%$ of boys older than 12 years were below the third percentile of height. ${ }^{41}$ A review of the literature reveals some disagreement regarding the age at which growth retardation occurs in children with TM. Some reports found that slowing of growth was more evident as puberty approached, ${ }^{9,40}$ while others noted a tendency for retarded growth at age 8 to 10 years. ${ }^{42}$ Recent study of Indian children with TM showed marked growth retardation in height and weight after age 11 years in boys and after age 9 years in girls. ${ }^{43}$ Data on Jordanian TM patients show that growth retardation worsened after the age of 10 years. ${ }^{20}$

\section{Orofacial Studies}

The orofacial disfigurements presented here are comparable to those earlier reported by Logothetis et $\mathrm{al}^{44}$ on Greek TM patients (mean age: $10 \pm 4$ years), showing that $32 \%$ had normal appearance, $23 \%$ had mild maxillary overgrowth, and 14\% displayed "rodent-like facies." Other features of TM are recently described in some large studies. In a study on 60 Thai thalassemic patients, Wisetsin ${ }^{45}$ showed that $63 \%$ had thickening of frontal bone, $73 \%$ had thin mandibular cortex, $8 \%$ had "hair-onend" calvarium, and 5\% had absence of maxillary sinus. In addition to genetic and racial influence, the considerable variation in the frequency of TM manifestations among different studies must be interpreted in the light of course of illness and management, sample size, and age.

\section{Morbidity and Mortality of TM}

The major causes of morbidity and mortality in TM are anemia and iron overload. Repeated blood transfusions represent the major cause of iron overload (transfusional hemosiderosis) in TM. Iron overload cardiomyopathy (myocardial siderosis) is considered a leading cause of morbidity and mortality in 63.6 to $71 \%$ of the TM patients. $6,8,46$ Infections constitute the second most common cause of morbidity and mortality in TM patients. Over the years, the significant advances in managing iron-induced cardiac disease; infections are becoming the leading cause of death in Western countries. ${ }^{3,6,23}$ Individuals who have not been regularly transfused usually die before the second or third decade. Survival of individuals who have been regularly transfused and treated with appropriate chelation extends beyond age of 40 years. ${ }^{6,23}$ Prognosis of TM has improved substantially in the last 20 years following recent medical advances in transfusion, drugs designed to remove excess iron (iron chelators), and bone marrow transplantation therapy. Early diagnosis and treatment of thalassemia is of paramount importance to limit the complications, as the pathological entity of TM increased with age.

\section{Dental Considerations}

Dental management of a TM patient requires special concern as this patient could suffer from complications of the disease, such as heart and liver dysfunction, diabetes, compromised immunity, and postsplenectomy infection. Careful assessment of the condition will avoid complications that could arise either as a result of the disease or due to treatment. Most patients with thalassemia can safely receive routine dental hygiene care and dental treatment. However, long procedures should be avoided in patients with more severe forms of the disease.

Prior to conducting an intensive dental procedure, a thorough medical history and current medical report are required for a basic health assessment. This should include: (1) Present Hb level; (2) iron chelators and other medicines received, such as antibiotics; (3) presence or history of splenectomy; (4) overall patient prognosis and life expectancy. Complex surgical treatment is contraindicated in patients if blood transfusion and chelator therapy are not under control. Close liaison 
with the hematology team is required to determine the potential complications when delivering an extensive dental treatment and the necessary measures should be put in place to confirm safe treatment.

Any invasive dental procedure in these patients should be done after transfusion where the $\mathrm{Hb}$ level is more than $10 \mathrm{gm} / \mathrm{dL}$. Antibiotic prophylaxis similar to that used for the prevention of bacterial endocarditis should be administered. That is, $50 \mathrm{mg} / \mathrm{kg}$ of amoxicillin (to a maximum dose of $2 \mathrm{gm}$ ) 1 hour prior to dental work. If the patient is allergic to penicillin, $20 \mathrm{mg} / \mathrm{kg}$ of clindamycin (to a maximum dose of $600 \mathrm{mg}$ ) is the alternative regimen. Some of the medications to be avoided are sulfa drugs, chloramphenicol, ciprofloxacin, doxycycline, and aspirin. Acetaminophen (Paracetamol) is a safe alternative to aspirin.

The radiographic indiscernible of the inferior dental (alveolar) canal landmarks in many TM patients should be considered in conducting surgical intervention in the area of mandibular posterior teeth. Such attention is required to avoid injury to the inferior dental nerve. Elective surgery, such as the removal of asymptomatic impacted teeth, should be avoided.

Thalassemic patients are at risk of viral hepatitis due to the blood transfusion from donors infected by hepatitis $\mathrm{C}$ virus. Therefore appropriate precautions should be taken by the dental team when these patients are to be treated. Effective blood donor screening techniques is required to prevent transmission of the virus.

\section{Dental Caries and Periodontal Disease}

Patients with TM are at risk for dental caries and periodontal disease. Therefore, patients should be maintained on intensive preventive program with regular follow-up. Effective preventive measures should be implemented to minimize the need for extensive dental procedures. The dentist should monitor preventive dental care at routine follow-up visits. A 3-month recall may be necessary in some cases. Oral hygiene instructions, diet counseling, and reinforcement should be provided. Preventive measures, including prophylaxis, fluoride application, and fissure sealant, should be implemented to minimize the need for extensive dental procedures. In children in whom manual dexterity is limited, the use of electric brushes is recommended. A professional fluoride application of $1.23 \%$ acidulated phosphate fluoride gel or fluoride varnish should be provided at 3 months intervals. Fluoride varnish application is the best choice for children under age 6 .

\section{Orthodontic Treatment}

Orthodontic treatment for the TM patient is strictly elective. These patients may have malocclusion or skeletal abnormalities. Correction of drifted maxillary anterior teeth and increased overjet should be undertaken to improve esthetics, reduce susceptibility to trauma, avoid gingival inflammation, and improve functional ability and child's self-esteem. It is recommended that orthodontic treatment be initiated as early as possible concentrating on preventive and interceptive approaches. Orthodontic appliances should be designed to prevent irritation and bacterial infections of soft tissues. Low forces should be applied since the cortical plates are thin and because of increased bone remolding. Hence, these patients should be followed more closely with shorter intervals between appointments as teeth move faster compared with the normal situation. Smaller tooth size, short spiky roots, and reduced dental arch dimensions in TM should be considered in planning orthodontic treatment. However, the disease process may compromise the outcome of the planned treatment.

\section{Anesthesia and Sedation}

Most patients with TM can be treated normally using local anesthetic supplemented if necessary with inhalation sedation. Because of the possibility of impairing local circulation, short procedure can be performed using anesthetic without vasoconstrictor. However, if the procedure takes a long time, $2 \%$ lidocaine with a vasoconstrictor 1/100,000 epinephrine is preferred. Nitrous oxide can be safely used as far as the oxygen concentration is $>50 \%$, the flow rate is high, and the patient is able to ventilate adequately. Oral sedation is an alternative to help decrease preoperative anxiety levels. Midazolam (Versed $^{\circledR}$, Dormicum $\left.^{\circledR}\right)$ is one of the commonly used oral sedation agents in children. When taken orally, it is rapidly absorbed in the gastrointestinal tract, produces its peak effect in a relatively shorter time of about 30 minutes, and has a short half-life of about 1.75 hours.

\section{General Anesthesia}

Patients with TM may present for elective or emergency surgery, such as therapeutic splenectomy, cholecystectomy, correction of facial deformities, and operative treatment of fractures. Because of anemia and hypoxia caused by the disease, $\mathrm{Hb}$ level should be optimized preoperatively and maintained at adequate levels with transfusion and blood-saving strategies. Careful airway assessment is of vital importance, as difficult intubation may be encountered in the presence of maxillary hypertrophy or tonsillar enlargement.

Bisphosphonates are pyrophosphate analogs. Because of their potent inhibitors of bone resorption, they are widely used for the management of TM-associated osteoporosis and fracture prevention. However, in recent 
years, there have been an increasing number of cases of bisphosphonate-related osteonecrosis of the jaw. ${ }^{47}$ This is characterized by transmucosal exposure of necrotic bone, associated with poor gum healing, numbness, and jaw pain. For patients already on bisphosphonates, restorative and nonsurgical treatments appear to be safe. For invasive surgical procedure, patients should be advised of the risk preoperatively and closely monitored postoperatively. ${ }^{48}$

\section{Psychological Adjustment}

Patients with thalassemia are vulnerable to psychological challenges, including depression and anxiety that can impact patient motivation and willingness to accept dental interventions. Due to underlying chronic anemia, thalassemic patients may experience fatigue, dizziness, shortness of breath, headache, and leg cramps. Psychological support with the goal of improving emotional health should be tailored to the patient's age and their tolerance of the planned procedure on the day of treatment. The appointment should be made as short as possible to reduce stress.

\section{Summary and Recommendations}

- Patients with TM are at high risk of dental caries and susceptible to periodontal disease. Effective preventive measures should be implemented to minimize the need for extensive dental procedures.

- Manifestations of TM increase with age. Early diagnosis and management allow more favorable prognosis and minimize complication.

- Reduction in size of tooth crown and dental arches in TM patients has an impact on the occlusal relationships. These changes should be taken into account when planning orthodontic treatment and orthognathic surgery.

- Pediatric dentists and orthodontists must be mindful of both the growth patterns associated with thalassemia and that delays in dental development have an impact on diagnosis and treatment timing.

- The radiographic absence of the inferior alveolar canal landmarks in many TM patients should be considered in conducting surgical intervention in the area of mandibular posterior teeth. Such attention is required to avoid injury to the inferior dental nerve.

- Dental management of a thalassemic patient requires special concern as this patient could suffer from complications of the disease, such as heart and liver dysfunction, diabetes, compromised immunity, and postsplenectomy infection.

- In a patient who has had a splenectomy, antibiotic prophylaxis similar to that used for the prevention of bacterial endocarditis should be used prior to invasive surgical procedure.

\section{REFERENCES}

1. Galanello R, Origa R. Beta-thalassemia. Orphanet J Rare Dis 2010 May;5:11.

2. Vichinsky EP. Changing patterns of thalassemia worldwide. Ann N Y Acad Sci 2005 Nov;1054:18-24.

3. Olivieri NF. The $\beta$-thalassemia. N Engl J Med 1999 Jul;341(2): 99-109.

4. Perrotta S, Cappellini MD, Bertoldo F, Servedio V, Iolascon G, D'Agruma L, Gasparini P, Siciliani MC, Iolascon A. Osteoporosis in $\beta$-thalassaemia major patients: analysis of the genetic background. Br J Haematol 2000 Nov;111(2):461-466.

5. Voskaridou E, Kyrtsonis M-C, Terpos E, Skordili M, Theodoropoulos I, Bergele A, Diamanti E, Kalovidouris A, Loutradi A, Loukopoulos D. Bone resorption is increased in young adults with thalassaemia major. Br J Haematol 2001 Jan;112(1):36-41.

6. Borgna-Pignatti C, Rugolotto S, De Stefano P, Zhao H, Cappellini MD, Del Vecchio GC, Romeo MA, Forni GL, Gamberini MR, Ghilardi R, et al. Survival and complications in patients with thalassemia major treated with transfusion and deferoxamine. Haematologica 2004 Oct;89(10):1187-1193.

7. Toumba M, Sergis A, Kanaris C, Skordis N. Endocrine complications in patients with thalassaemia major. Pediatr Endocrinol Rev 2007 Dec;5(2):642-648.

8. Origa R, Galanello R. Pathophysiology of beta thalassaemia. Pediatr Endocrinol Rev 2011 Mar;8(Suppl 2):S263-S270.

9. Borgna-Pignatti C, DeStefano P, Zonta L, Vullo C, DeSanctis V, Melevendi C, Naselli A, Masera G, Terzoli S, Gabutti V, et al. Growth and sexual maturation in thalassemia major. J Pediatr 1985 Jan;106(1):150-155.

10. Modell B. Management of thalassaemia major. Br Med Bull 1976 Sep;32(3):270-276.

11. Haidar R, Musallam KM, Taher AT. Bone disease and skeletal complications in patients with $\beta$-thalassemia major. Bone 2011 Mar;48(3):425-432.

12. Demirjian A, Goldstein H, Tanner JM. A new system of dental age assessment. Hum Biol 1973 May;45(2):211-227.

13. Hattab FN, Hazza'a AM, Yassin OM, Daoud N. Caries risk in patients with thalassaemia major. Int Dent J 2001 Feb;51(1): 35-38.

14. Hattab FN. Periodontal condition and orofacial changes in patients with thalassemia major: a clinical and radiographic overview. J Clin Pediatr Dent 2012 Spring;36(3):301-307.

15. Hattab FN, Qudeimat MA, Al-Rimawi HS. Dental discoloration: an overview. J Esthet Dent 1999 Nov;11(6):291-310.

16. Hattab FN, Abu Alhaija ESJ, Yassin OM. Tooth crown size of the permanent dentition in subjects with thalassemia major. Dent Anthrop 2000;14:7-13.

17. Hattab FN. Mesiodistal crown diameters and tooth size discrepancy of permanent dentition in thalassemic patients. J Clin Exp Dent 2013 Dec;5(5):e239-e244.

18. Hattab FN, Yassin OM. Dental arch dimensions in subjects with beta-thalassemia major. J Contemp Dent Pract 2011 Nov;12(6):429-433.

19. Abu Alhaija ESJ, Hattab FN. Cephalometric measurements and facial deformities in subjects with $\beta$-thalassemia major. Eur J Orthod 2002 Feb;24(1):9-19.

20. Hattab FN. Patterns of physical growth and dental development in Jordanian children and adolescents with thalassemia major. J Oral Sci 2013 Feb;55(1):71-77. 
21. Sayyedi A, Pourdanesh F, Sarkari B, Nabavizadeh SH. Evaluation of oro-maxillofacial changes in major thalassemia. Inside Dent 2008 Feb;4(2).

22. Elangovan A, Mungara J, Joseph E, Guptha V. Prevalence of dentofacial abnormalities in children and adolescents with $\beta$-thalassaemia major. Indian J Dent Res 2013 Jul-Aug;24(4): 406-410.

23. Modell B, Khan M, Darlison M, Westwood MA, Ingram D, Pennell DJ. Improved survival of thalassaemia major in the UK and relation to $\mathrm{T}^{*}$ cardiovascular magnetic resonance. J Cardiovasc Magn Reson 2008 Sep;10(1):42.

24. Papamanthos MK, Varitimidis SE, Dailiana ZH, Kogia EI, Malizos KN. Computer-assisted evaluation of mandibular cortical width (MCW) index as an indicator of osteoporosis. Hippokratia 2014 Jul-Sep;18(3):251-257.

25. Parlani S, Nair P, Agrawal S, Beohar G, Katar U. Role of panoramic radiographs in the detection of osteoporosis. Oral Hyg Health 2014 Feb;2(1):121-124.

26. Hazza'a AM, Al-Jamal G. Radiographic features of the jaws and teeth in thalassaemia major. Dentomaxillofac Radiol 2006 Jul;35(4):283-288.

27. Van Dis ML, Langlais RP. The thalassemias: oral manifestations and complications. Oral Surg Oral Med Oral Pathol 1986 Aug;62(2):229-233.

28. Ja'afar MN, Al-Aswad FD. Oro-facial manifestations, microbial study and salivary enzyme analysis in patients with $\beta$-thalassemia major. J Bagh College Dent 2012;24(1):52-56.

29. Abdulla HI, Hasen OM. Orofacial structural changes in Iraqi patients with $\beta$-thalassemia major. Iraqi Dent J 2014;36(2): 76-82.

30. Pedullà E, Scibilia M, Saladdino G, Colletta G, Rapisarda $S$. Dental and periodontal condition in patients affected by $\beta$-thalassemia major and $\beta$-thalassemia intermedia: a study among adults in Sicily, Italy. J Dent Health Oral Disord Ther 2015 Nov;3(1):81-85.

31. Leonardi R, Verzì P, Caltabiano M. Epidemiological survey of the prevalence of dental caries in young thalassemia major patients. Stomatol Mediterr 1990 Apr-Jun;10(2):133-136.

32. De Mattia D, Pettini PL, Sabato V, Rubini G, Laforgia A, Schettini F. Oromaxillofacial changes in thalassemia major. Minerva Pediatr 1996 Jan-Feb;48(1-2):11-20.

33. World Health Organization. Preventive methods and programs for oral diseases. Technical report series 713, Geneva. 1984.

34. Siamopoulou-Mavridou A, Mavridis A, Galanakis E, VasakosS, Fatourou H, Lapatsanis P. Flow rate and chemistry of parotid saliva related to dental caries and gingivitis in patients with thalassaemia major. Int J Paediatr Dent 1992 Aug;2(2): 93-97.

35. Lugliè PF, Campus G, Deiola C, Mela MG, Gallisai D. Oral condition, chemistry of saliva, and salivary levels of Streptococcus mutans in thalassemic patients. Clin Oral Investig 2002 Dec;6(4):223-226.

36. Wactawski-Wende J. Periodontal diseases and osteoporosis: association and mechanisms. Ann Periodontol 2001 Dec;6(1):197-208.

37. Garn SM, Osborne RH, McCabe KD. The effect of prenatal on crown dimensions. Am J Phys Anthropol 1979 Nov;51(4): 665-677.

38. Scutellari PN, Orzincolo C, Andraghetti D, Gamberini MR. Anomalies of masticatory apparatus in beta-thalassemia. The present status after transfusion and iron-chelating therapy. Radiol Med 1994 Apr;87(4):389-396.

39. Demirjian A, Buschang PH, Tanguay R, Patterson DK. Interrelationships among measures of somatic, skeletal, dental and sexual maturity. Am J Orthod 1985 Nov;88(5):433-438.

40. Kattamis C, Liakopoulou T, Kattamis A. Growth and development in children with thalassemia major. Acta Paediatr Scand 1990 Mar;366(Suppl):111-117.

41. Kwan EY, Lee AC, Li AM, Tam SC, Chan CF, Lau YL, Low LC. A cross-sectional study of growth, puberty and endocrine function in patients with thalassaemia major in Hong Kong. J Paediatr Child Health 1995 Apr;31(2):83-87.

42. Yesilipek MA, Bircan I, Oygür N, Ertug H, Yegin O, Güven AG. Growth and sexual maturation in children with thalassemia. Haematologica 1993 Jan-Feb;78(1):30-33.

43. Saxena A. Growth retardation in thalassemia major patients. Int J Hum Genet 2003 Jan;3(4):237-246.

44. Logothetis J, Economidou J, Constantoulakis M, AugoustakiO, Loewenson RB, Bilek M. Cephalofacial deformities in thalassemia major (Cooley's anemia). Am J Dis Child 1971 Apr;121(4):300-306.

45. Wisetsin S. Cephalography in thalassemic patients. J Dent Assoc Thai 1990 Nov-Dec;40(6):260-268.

46. Wood JC. Cardiac complications in thalassemia major. Hemoglobin 2009;33(Suppl 1):S81-S86.

47. Fedele S, Kumar N, Davies R, Fiske J, Greening S, Porter S. Dental management of patients at risk of osteochemonecrosis of the jaws: a critical review. Oral Dis 2009 Nov;15(8):527-537.

48. Kumar, N.;Hattab, FN. Dental care. In:Cappellini MD, Cohen A, Porter J, Taher A, Viprakasit V, editors. Guidelines for the Management of Transfusion Dependent Thalassaemia (TDT). 3rd ed. Chapter 11. Nicosia (Cyprus): Thalassaemia International Federation (TIF Publication No. 20); 2014. p. 178-184. 